\title{
Rrp9 as a Potential Novel Antifungal Target in Candida albicans: Evidences from In Silico Studies
}

\author{
Awad Ali, Archana Wakharde and Sankunny Mohan Karuppayil*
}

School of Life Sciences (DST-FIST\&UGC-SAP Sponsored), S.R.T.M. University (NAAC Accredited with "A" grade), Nanded, 431606, Maharashtra state, India

*Corresponding author: Sankunny Mohan Karuppayil, Professor and Director, School of Life Sciences (DST-FIST\&UGC-SAP Sponsored), S.R.T.M. University (NAAC Accredited with "A" grade), Nanded, 431606, Maharashtra state, India, Tel: +919764386253; E-mail: prof.karuppayil@gmail.com

Received date: November 06, 2017; Accepted date: November 22, 2017; Published date: November 30, 2017

Citation: Ali A, Wakharde A, Karuppayil SM (2017) Rrp9 as a Potential Novel Antifungal Target in Candida albicans: Evidences from In Silico Studies. Med Mycol Open Access Vol.3 No. 2: 26.

Copyright: (C2017 Ali A, et al. This is an open-access article distributed under the terms of the Creative Commons Attribution License, which permits unrestricted use, distribution, and reproduction in any medium, provided the original author and source are credited.

\section{Abstract}

Dicyclomine is a selective muscarinic M1 receptor antagonist in humans. Dicyclomine targets signal transduction genes and inhibits virulence factors in the human pathogen, Candida albicans. Muscarinic acetylcholine receptors are not known to exist in $C$. albicans. We carried out a search to identify a muscarinic receptor like proteins in C. albicans. A BLAST protein analysis revealed that a C. albicans protein Rrp9 shares $54 \%$ identity and $71 \%$ similarity with human muscarinic M1 receptor at 24 amino acids overlap. Global alignment between human muscarinic M1 receptor and $C$. albicans Rrp9 showed $19 \%$ identity and $33 \%$ similarity at 570 amino acid residues overlap. Our molecular docking study of dicyclomine with C. albicans, Rrp9, Gpr1, Ste2 and Ste3 showed that dicyclomine could bind only with Rrp9 by forming hydrogen bond interactions with VAL386 and ILE313 amino acid residues in the active site region. Dicyclomine bound with human muscarinic receptor M1 by forming hydrogen bond interaction with ARG123 at the active site region. Our study suggests that Rrp9 may be considered as a potential target for dicyclomine in $C$. albicans.

Keywords: Candida albicans Rrp9; Candida albicans Gpr1; Candida albicans Ste2; Candida albicans ste3; Human muscarinic receptor M1; Molecular Docking; In Silico; Novel antifungal target

\section{Introduction}

Muscarinic acetylcholine receptors are typical G-protein coupled receptors which can permit signal transmission through heterometric G-protein to downstream second messengers. Five types of muscarinic receptors (M1-M5) are reported in humans that carry out many physiological roles $[1,2,3]$. Dicyclomine is a human muscarinic receptor antagonist targeting $\mathrm{M} 1$ and incline to target M3. Even though muscarinic receptors like proteins are not reported in Candida albicans, GPCR proteins are reported.
Human G-protein coupled receptors (GPCRs) are complex compared to $C$. albicans GPCR. C. albicans has GPCR proteins such as Gpr1, Ste2, and Ste3. Gpr1 is a glucose sensor that plays important roles in morphogenesis [4]. Ste2 and Ste3 are pheromone receptors which play roles in mating and morphogenesis [5]. Our earlier study reported that dicyclomine can target signal transduction genes and inhibit the virulence factors in C. albicans [6]. In this study, we have tried to identify a muscarinic $\mathrm{M} 1$ receptor like protein in C. albicans using an In silico approach.

\section{Materials and Methods}

\section{Basic local alignment search tool protein (BLASTp)}

The sequence of the human muscarinic receptors M1 was retrieved from Uniprot public domain protein database. FASTA sequence of muscarinic acetylcholine receptors M1 compared with Candida albicans proteins using a BLAST protein search. The primary sequences of $C$. albicans Gpr1, Ste2, and Ste3 were retrieved in FASTA format from Uniprot public domain protein database with accession numbers of A0A1D8PN42, A0A1D8PTB4, and Q5A9Z4 respectively. The sequences between muscarinic receptors $\mathrm{M} 1$ and C. albicans GPCR proteins (Gpr1, Ste2, and Ste3) were analyzed using a BLASTp.

\section{Global alignment}

The Global alignment between Rrp9 (Q5A213), Gpr1 (A0A1D8PN42), Ste2 (A0A1D8PTB4), and Ste3 (Q5A9Z4) from $C$. albicans and human muscarinic receptor M1 (P11229) was done by using Geneious software server from Biomatters, Auckland, New Zealand [7].

\section{Homology modeling of protein sequences}

The retrieved sequences of $C$. albicans Rrp9, Gpr1, Ste2, and Ste3 were submitted to Phyre 2 homology modeling server for modeling of three dimensional structures of proteins [8]. Tertiary structure predictions were done by using Phyre $2^{\circledR}$ (Figure 1) and validation of the tertiary structures were done 
through Procheck [9]. These predicted structures of Rrp9, Gpr1, Ste2, and Ste3 were used for docking studies [10].

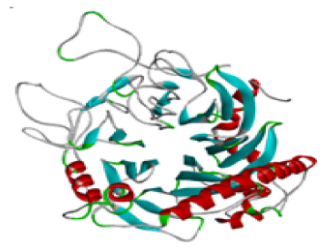

Rup9

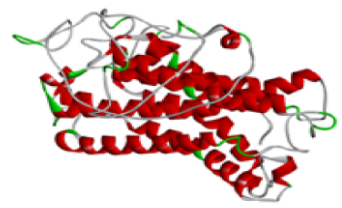

Ste3

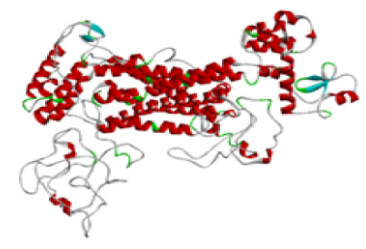

Gpr1

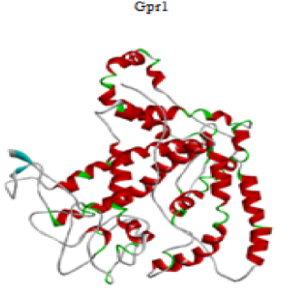

Ste2
Figure 1: Predicted structures of Candida albicans Rrp9, Gpr1, Ste3, and Ste2 by using Phyre 2 server.

\section{D structure of muscarinic receptor M1}

The 3D structure of muscarinic receptor $M 1$ was obtained from Protein Data Bank (RCSB PDB) with PDB ID, 5CXV. The previous ligands on $5 C X V$ PDB structure were removed by using Discovery Studio (DS) 4.1 Visualizer [10,11].

\section{Ligand preparation}

Dicyclomine Structure was retrieved from Pubchem database in SDF format. The chemical structure followed by $2 \mathrm{D}$ structure cleaning, 3D optimization and viewing is done by using MarwinView and saved in Mol2 file format. Dicyclomine Mol2 file was converted to PDBQT file format in Autodock Tools version 1.5.6rc2 [10].

\section{Molecular docking}

The Autodock Tools package version 1.5.6rc2 was employed to generate docking input files. All the nonpolar hydrogens were merged and the water molecules were removed. For Docking, a grid spacing of $0.375 \AA$ and $60 \times 60 \times 60$ number of points was used. Before docking all the water molecules were removed from the protein structures followed by addition of Hydrogen atoms to receptors and merging non-polar hydrogens. Modeled three dimensional structures of C. albicans Rrp9, Gpr1, Ste2, and Ste3 and the 3D structure of human muscarinic receptor M1 as well as structure of the ligand, dicyclomine were converted to PDBQT format [10]. Molecular docking study of dicyclomine with C. albicans Rrp9, Gpr1, Ste2, Ste3, and muscarinic M1 receptor were carried out by using AutoDock ${ }^{\circledast}$ suite as molecular-docking tool [12]. Default optimization parameters were done using Lamarckian Genetic Algorithm with a population size of 150 dockings. Autodock ${ }^{\circledR}$ tools generated sixty possible binding conformations, i.e. sixty runs for each docking by using Genetic Algorithm (GALS) searches. The grid box used for specifying the search space was set at $60 \times 60 \times 60$ centred on proteins with a default grid point spacing of $0.375 \AA$. Autogrid was used to obtain pre calculated grid maps. After completion of docking, most suitable conformation was chosen based on lowest docked energy. Selected conformations were analyzed by Autodock tool [10].

\section{Analysis of molecular docking}

The ligand-protein complex preparation between dicyclomine DLG file and protein structures of Rrp9, Gpr1, Ste2, Ste3 and muscarinic M1 receptor in PDBQT format was done using AutoDock 1.5.6rc2. Docked structures were saved in PDBQT format and analyzed for amino acid residues in the ligand binding sites of the proteins structure associated with the binding interactions such as hydrogen bonding. This analysis was visualized by using Discovery Studio 4.1 Visualizer software [10].

\section{Results}

\section{Muscarinic acetylcholine M1 receptor like protein in Candida albicans}

Human muscarinic receptor M1 exhibited 54\% identity and $71 \%$ similarity with $C$. albicans Rrp9 protein at an overlap of 24 amino acids (Table 1). Global alignment between human muscarinic M1 receptor and C. albicans Rrp9 protein showed that muscarinic receptor $\mathrm{M} 1$ has $19 \%$ identity and $33 \%$ similarity with C. albicans Rrp9 at the amino acid level at an overlap of 570 amino acids (Table 1) (Figure 2a). A BLASTp and global alignment analysis between human muscarinic $\mathrm{M} 1$ receptor and $C$. albicans GPCR proteins, Gpr1, Ste2, and Ste3 presented various identities and similarities at amino acids overlap. C. albicans Gpr1 showed $16 \%$ identity and $28 \%$ similarity with human muscarinic M1 receptor at 828 amino acids overlap (Table 1) (Figure 2b). C. albicans Ste2 exhibited $16 \%$ identity and $30 \%$ similarity with human muscarinic M1 receptor at 517 amino acids overlap (Table 1) (Figure 2c). C. albicans Ste3 also showed 19\% identity and 32\% similarity with human muscarinic M1 receptor at 505 amino acid residues overlap (Table 1 ) (Figure $2 d$ ).

Table 1: BLAST protein search and Global alignment between Candida albicans proteins, Rrp9, Gpr1, Ste2, Ste3 and Human muscarinic acetylcholine receptor M1 (CHRM1).

\begin{tabular}{|l|l|l|l|l|l|l|}
\hline \multirow{2}{*}{ Human } & \multicolumn{2}{|l|}{ BLASTp } & \multicolumn{2}{l|}{ Global alignment } \\
\cline { 2 - 5 } & \begin{tabular}{l} 
C. $\begin{array}{l}\text { C. albicans } \\
\text { proteins }\end{array}$ \\
\cline { 2 - 5 }
\end{tabular} & Identity $\%$ & A.A. overlap & Identity $\% \quad$ Similarity $\%$ \\
\hline
\end{tabular}




\begin{tabular}{|l|l|l|l|l|l|l|l|}
\hline \multirow{3}{*}{ CHRM1 } & Rrp9 & 54 & 71 & 24 & 19 & 33 & 570 \\
\cline { 2 - 8 } & Gpr1 & 39 & 57 & 28 & 16 & 28 & 828 \\
\cline { 2 - 8 } & Ste2 & 35 & 54 & 37 & 16 & 19 & 30 \\
\cline { 2 - 8 } & Ste3 & 46 & 66 & 15 & 32 & 505 \\
\hline
\end{tabular}

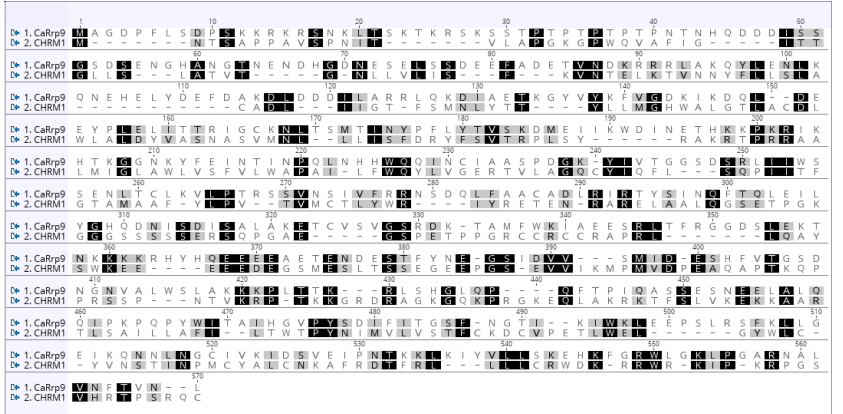

Figure 2a: Pairwise alignment between amino acid sequences of Candida albicans Rrp9 (CaRrp9) and Human muscarinic acetylcholine receptor M1 (CHRM1) showing 19\% identity and $33 \%$ similarity. Black color boxes indicate identical residues and gray color boxes indicate similar residues.

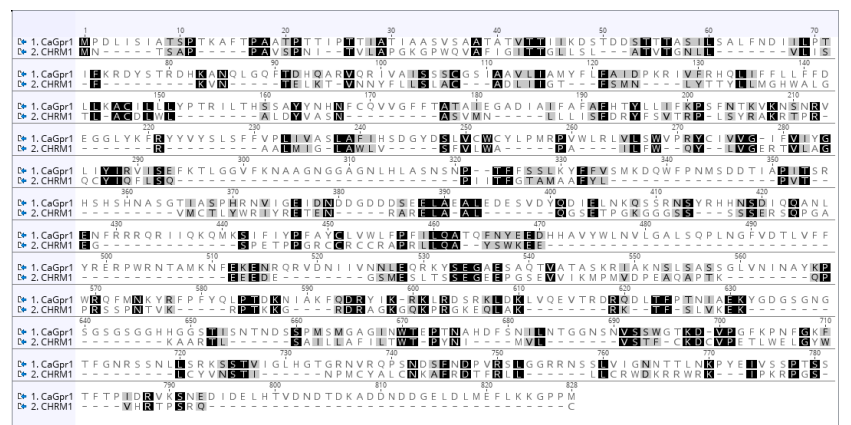

Figure 2b: Pairwise alignment between amino acid sequences of Candida albicans Gpr1 (CaGpr1) and Human muscarinic acetylcholine receptor M1 (CHRM1) showing 16\% identity and $28 \%$ similarity. Black color boxes indicate identical residues and gray color boxes indicate similar residues.

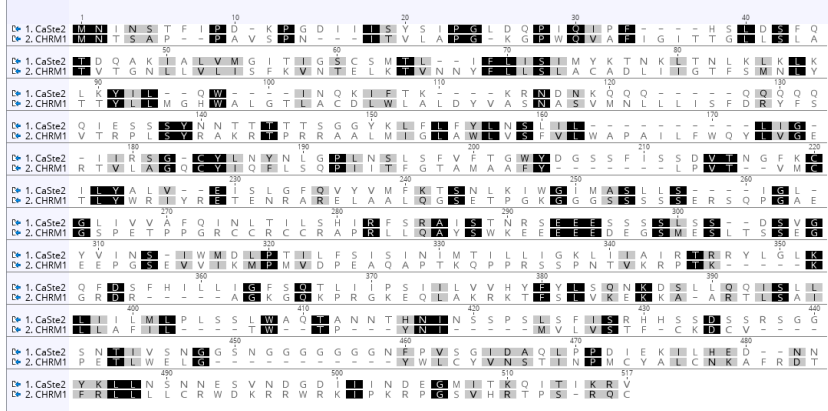

Figure 2c: Pairwise alignment between amino acid sequences of Candida albicans Ste2 (CaSte2) and Human muscarinic acetylcholine receptor M1 (CHRM1) showing 16\% identity and $30 \%$ similarity. Black color boxes indicate identical residues and gray color boxes indicate similar residues.

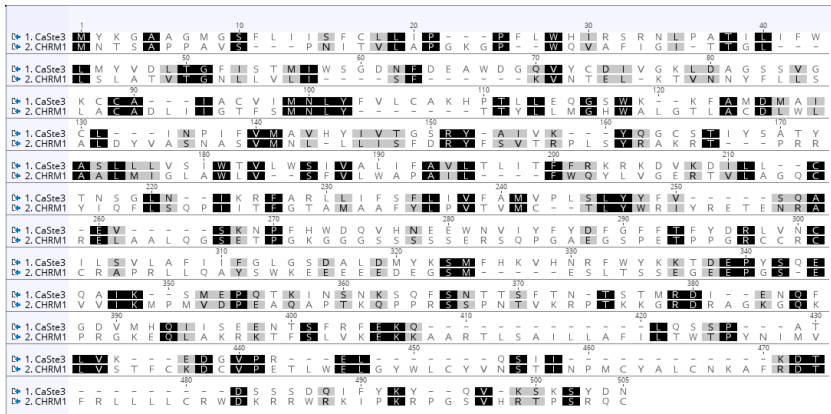

Figure 2d: Pairwise alignment between amino acid sequences of Candida albicans Ste3 (CaSte3) and Human muscarinic acetylcholine receptor M1 (CHRM1) showing 19\% identity and $32 \%$ similarity. Black color boxes indicate identical residues and gray color boxes indicate similar residues.

\section{Dicyclomine docks with Rrp9, Gpr1, Ste2, Ste3 and} muscarinic receptor $\mathrm{M} 1$

Dicyclomine was found to bind with C. albicans Rrp9, Gpr1, Ste2, and Ste3 with binding energies of $-9.32,-6.50,-8.73$, and $-8.43 \mathrm{Kcal} / \mathrm{mol}^{-1}$ respectively (Table 2 ). Dicyclomine bound with Rrp9 by forming hydrogen bond interaction with VAL386 and ILE313 amino acid residues in the active site region (Table 2) (Figure 3a). Dicyclomine did not form any hydrogen bond interactions with $C$. albicans, Gpr1, Ste2, and Ste3 (Table 2) (Figures $3 b-3 d$ ). Dicyclomine showed binding with muscarinic receptor $\mathrm{M} 1$ by binding energy of $-6.84 \mathrm{Kcal} / \mathrm{mol}^{-1}$ (Table 2). It formed hydrogen bonding with ARG123 amino acid residue in 
the active site region of muscarinic receptor M1 (Table 2) (Figure 3e).

Table 2: Molecular interactions of dicyclomine with Candida albicans proteins, Rrp9, Gpr1, Ste2, Ste3 and human muscarinic acetylcholine receptor M1 (CHRM1).

\begin{tabular}{|c|c|c|c|c|c|c|c|}
\hline Ligand & Receptor & $\begin{array}{l}\text { Run } \\
\text { no. }\end{array}$ & $\begin{array}{l}\text { Interacting } \\
\text { residues }\end{array}$ & $\begin{array}{l}\text { Interacting atoms } \\
\text { (Amino acid .. } \\
\text { ligand }\end{array}$ & $\begin{array}{l}\text { H bond } \\
\text { formed }\end{array}$ & $\begin{array}{l}\text { Binding energy } \\
\text { (Kcal/mol) }\end{array}$ & Electrostatic energy \\
\hline \multirow[t]{6}{*}{ Dicyclomine } & CaRrp9 & 31 & VAL386 & O -------- O1 & 2 & -9.32 & -0.11 \\
\hline & & & ILE313 & HN ---- O2 & & & \\
\hline & CaGpr1 & 37 & ---- & ---- & ---- & -6.50 & -0.04 \\
\hline & CaSte2 & 51 & ---- & ---- & ---- & -8.73 & 0.02 \\
\hline & CaSte3 & 40 & ---- & ----- & ---- & -8.43 & -0.01 \\
\hline & CHRM1 & 27 & ARG123 & HH21 --- O2 & 1 & -6.84 & -0.15 \\
\hline
\end{tabular}

\section{Discussion}

The human muscarinic M1 receptor antagonist, dicyclomine is reported to inhibit Candida albicans growth and virulence factors [6]. In this study, we found that $C$. albicans Rrp9 shares identity and similarity with the human muscarinic receptor M1. Rrp9 has 19\% identity and 33\% similarity with human muscarinic receptor $\mathrm{M} 1$ at 570 amino acid residues overlap (Table 1) (Figure 2a). Also, human muscarinic $M 1$ receptor exhibited identities and similarities with $C$. albicans GPCR proteins, Gpr1, Ste2, and Ste3. C. albicans Gpr1 showed $16 \%$ identity and $28 \%$ similarity with muscarinic receptor M1 at an overlap of 828 amino acid residues (Table 1) (Figure 2b). C. albicans Ste2 exhibited $16 \%$ identity and $30 \%$ similarity with human muscarinic $\mathrm{M} 1$ receptor at 517 amino acid residues overlap (Table 1) (Figure 2c) while $C$. albicans Ste3 presented $19 \%$ and $32 \%$ similarity with muscarinic M1 receptor at an overlap of 505 amino acid residues (Table 1) (Figure 2c). But our molecular docking study showed that dicyclomine could bind only with Rrp9 and not with Gpr1, Ste2, and Ste3. Dicyclomine formed hydrogen bond interactions with VAL386 and ILE313 amino acid residues in the active site region of Rrp9 (Table 2) (Figure 3a). Even though dicyclomine had good binding energies with Gpr1, Ste2, and Ste3 it did not form any hydrogen bond interactions with them (Table 2) (Figures 3b-3d). Dicyclomine could bind with human muscarinic receptor M1 by forming hydrogen bond interaction to ARG123 amino acid residue at the active site region (Table 2) (Figure $3 \mathrm{e}$ ). $C$. albicans Rrp9 protein is a U3 small nucleolar ribonucleoproteinassociated protein that is involved in pre-ribosomal RNA processing and it has roles in cellular response to certain drugs $[13,14]$. Rashki et al. reported changes in the expression of more than 1000 genes during yeast to hyphal form transition using a cDNA microarray study. They found 2.05 fold increases in the expression of CaRrp9 during yeast to hyphal form transition [15]. We hypothesis that binding of dicyclomine with Rrp9 may affect its functions causing inhibition of $C$. albicans growth, dimorphism, and biofilm formation. Our study suggests that Rrp9 protein may be considered as a potential novel antifungal target for dicyclomine in C. albicans.

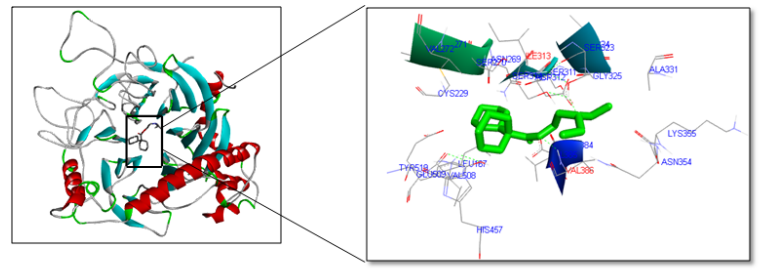

Figure 3a: Molecular interactions of dicyclomine with the active site pocket residues of modeled structure of Candida albicans Rrp9 protein. The green dotted line represents the hydrogen bond formation and red letters show the amino acid residues involved in the bonding and dicyclomine.

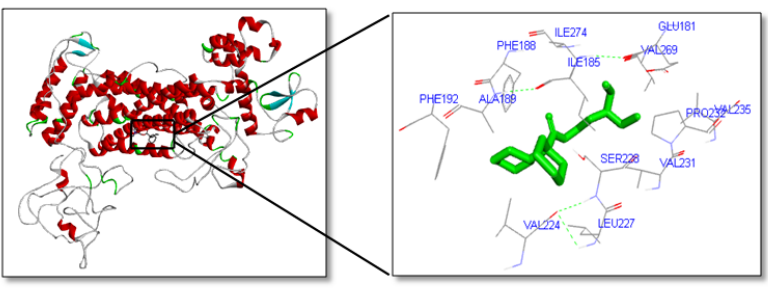

Figure 3b: Molecular interactions of dicyclomine with the active site pocket residues of modeled structure of Candida albicans Gpr1 protein. It shows no green dotted line indicating that dicyclomine does not form hydrogen bond interaction with Gpr1. 


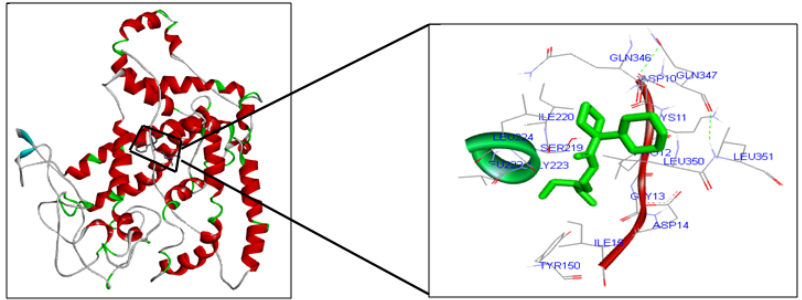

Figure 3c: Molecular interactions of dicyclomine with the active site pocket residues of modeled structure of Candida albicans Ste 2 protein. It shows no green dotted line indicating that dicyclomine does not form hydrogen bond interaction with Ste2.

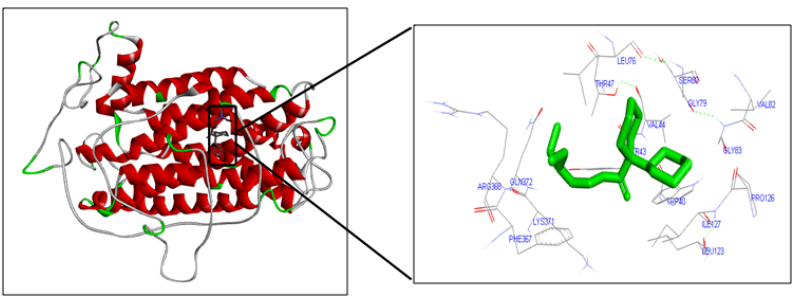

Figure 3d: Molecular interactions of dicyclomine with the active site pocket residues of modeled structure of Candida albicans Ste3 protein. It shows no green dotted line indicating that dicyclomine does not form hydrogen bond interaction with Ste3.

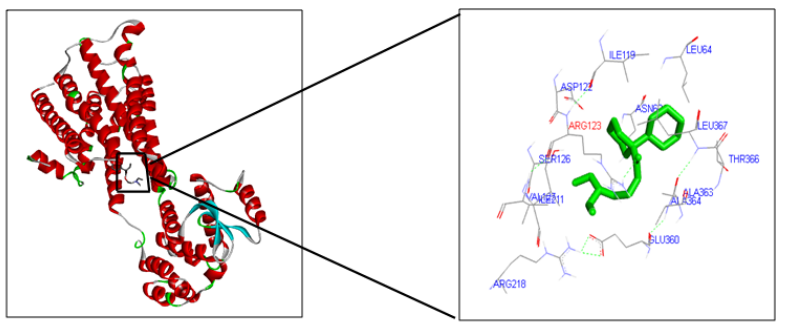

Figure 3e: Molecular interactions of dicyclomine with the active site pocket residues of Human muscarinic acetylcholine M1 receptor (CHRM1). The green dotted line represents the hydrogen bond formation and red letters show the amino acid residue involved in the bonding and dicyclomine.

\section{Acknowledgment}

AA and SMK are thankful to Prof. P. Vidyasagar, Vicechancellor, S.R.T.M. University, Nanded, Maharashtra for the facilities. SMK is also thankful to UGC SAP \& DAT FIST for infrastructural support.

\section{References}

1. Hulme EC, Birdsall NJM, Buckley NJ (1990) Muscarinic receptor subtypes. Ann Rev Pharm Toxico 30: 633-673.

2. Caulfield MP (1993) Muscarinic receptors-characterization, coupling and function. Pharmacol Ther 58: 319-379.

3. Caulfield MP, Birdsall NJ (1998) International Union of Pharmacology. XVII. Classification of muscarinic acetylcholine receptors. Pharmacol Rev 50: 279-290.

4. Versele M, Lemaire K, Thevelein JM (2001) Sex and sugar in yeast: two distinct GPCR systems. EMBO Rep 2: 574-579.

5. Bahn YS, Xue C, Idnurm A, Rutherford JC, Heitman J, et al. (2007) Sensing the environment: lessons from fungi. Natu Rev Micro 5: 57-70.

6. Awad Ali, Jadhav A, Jangid P, Patil R, Shelar A, et al. (2017) The human muscarinic acetylcholine receptor antagonist, Dicyclomine targets signal transduction genes and inhibits the virulence factors in the human pathogen, Candida albicans. Antibiotics, Under Review.

7. Kearse $M$, Moir R, Wilson A, Stones-Havas S, Cheung $M$, et al (2012) Geneious Basic: an integrated and extendable desktop software platform for the organization and analysis of sequence data. Bioinformatics 28: 1647-1649.

8. Kelley LA, Mezulis S, Yates CM, Wass MN, Sternberg MJ, et al. (2015) The Phyre2 web portal for protein modeling, prediction and analysis. Natu Protoc 10: 845-858.

9. Laskowski RA, MacArthur MW, Thornton JM (2001) PROCHECK: validation of protein structure coordinates. Volume F, Crystall Biol Macrom pp: 722-725.

10. Kumar A, Bora U (2014) Molecular docking studies of curcumin natural derivatives with DNA topoisomerase I and II-DNA complexes. Interdiscip Sci 6: 285

11. Baby ST, Sharma S, Enaganti S, Cherian PR (2016) Molecular docking and pharmacophore studies of heterocyclic compounds as Heat shock protein 90 (Hsp90) Inhibitors. Bioinformation 12: 149.

12. Morris GM, Goodsell DS, Halliday RS, Huey R, Hart WE, et al. (1998) Automated docking using a Lamarckian genetic algorithm and an empirical binding free energy function. J Comput Chem 19: 1639-1662.

13. Enjalbert B, Moran GP, Vaughan C, Yeomans T, MacCallum DM, et al. (2009) Genome-wide gene expression profiling and a forward genetic screen show that differential expression of the sodium ion transporter Ena21 contributes to the differential tolerance of Candida albicans and Candida dubliniensis to osmotic stress. Mol Microbiol 72: 216-228.

14. Aoyama T, Nakayama H, Ueno K, Inukai T, Tanabe K, et al. (2014) Genome-wide survey of transcriptional initiation in the pathogenic fungus, Candida glabrata. Genes Cells 9: 478-503.

15. Rashki A, Ghalehnoo ZR, Dominguez A (2012) The early response of Candida albicans filament induction is coupled with wholesale expression of the translation machinery. Compar Clini Path 21: 1533-1545. 